\title{
FAQ für schwangere Frauen und ihre Familien zu spezifischen Risiken der COVID-19-Virus-Infektion
}

Hinweise und „Frequently Asked Questions" (FAQ) vom Berufsverband der Frauenärzte e.V. (BVF) und der Deutschen Gesellschaft für Gynäkologie und Geburtshilfe e.V. (DGGG) vereint im German Board and College of Obstetrics and Gynecology (GBCOG).

Bin ich als Schwangere durch das Coronavirus gefährdeter als andere Frauen?

Zum gegenwärtigen Zeitpunkt gibt es international keinen Hinweis, dass Schwangere durch das neuartige Coronavirus (SARS-CoV-2) gefährdeter sind als die allgemeine Bevölkerung. Es wird erwartet, dass die große Mehrheit der schwangeren Frauen nur leichte oder mittelschwere Symptome, ähnlich einer Erkältung beziehungsweise Grippe aufweist. Schwerwiegendere Symptome wie Lungenentzündung scheinen bei älteren Menschen, Menschen mit geschwächtem Immunsystem oder Langzeiterkrankungen häufiger zu sein. Wenn eine bereits vor der Schwangerschaft bestehende Herz- oder Lungenerkrankung vorliegt, könnten eher Komplikationen bei Atemwegsinfektion wie bei der Coronavirusinfektion eintreten.

Welche Auswirkungen hat das Coronavirus auf mein Baby, wenn bei mir eine Infektion diagnostiziert wird?

Da es sich um ein sehr neues Virus handelt, fangen wir gerade erst an, etwas darüber zu lernen. Es gibt keine Hinweise auf ein erhöhtes Risiko für Fehlgeburten. Es gibt auch keine Hinweise darauf, dass das Virus während der Schwangerschaft auf das Baby übertragen werden kann (dies wird als vertikale Übertragung bezeichnet). Es wird daher als unwahrscheinlich angesehen, dass das Virus beim Fetus zu Anomalien führt. Bislang wurde international nur über 20 Schwangerschaften berichtet, alle fanden in China statt. In keinem Fall war ein Neugeborenes infiziert. Es wurden keine Auffälligkeiten bei Mutter und Kind berichtet.

Was kann ich tun, um mein Risiko, am Coronavirus zu erkranken, zu verringern? Das Wichtigste ist, dass Sie Ihre Hände regelmäßig und effektiv waschen, sobald Sie von öffentlichen Orten nach Hause oder an den Arbeitsplatz kommen. Vermeiden Sie unbedingt den Kontakt mit allen Personen, auch der Familie, wenn diese Symptome einer Erkältungskrankheit oder Fieber aufweisen. Hier finden Sie nützliche Tipps, wie Sie das Infektionsrisiko am besten reduzieren können:

- https://www.bundesgesundheitsministerium.de/coronavirus.html

- https://www.infektionsschutz.de/cv/

- https://www.infektionsschutz.de/ hygienetipps/

Welche Reisehinweise gelten, wenn ich schwanger bin?

Prinzipiell gilt, dass Sie zur Vermeidung einer Übertragung Abstand zu Mitreisenden halten sollten - im Alltag, wie auch unterwegs in Bahnen, Bussen etc. Wenn Sie sich in Deutschland befinden, sollten Sie den
Ratschlägen des Bundesministeriums für Gesundheit folgen (www.bundesgesundheitsministerium.de), die regelmäßig entsprechend der sich entwickelnden Situation gemeinsam mit weiteren Bundesministerien und dem RKI aktualisiert werden. Alle Personen, einschließlich schwangerer Frauen, sollten vor Reiseantritt sicherstellen, dass sie über angemessene Versicherungsvereinbarungen verfügen. Sie sollten auch überprüfen, ob Ihre Reiseversicherung die Geburt und Betreuung Ihres Neugeborenen abdeckt, für den Fall, dass Sie im Ausland gebären sollten. Bezogen auf das Ausland ist das Reiserisiko für Schwangere nicht höher ist als für andere Reisende. Beachten Sie bitte die Seite des Auswärtigen Amts mit Reisewarnungen für bestimmte Gebiete (www.auswaertiges-amt.de).

Was gilt, wenn ich engen Kontakt zu Personen hatte, die auf COVID-19 positiv getestet wurden?

Eine Corona-Infektion äußert sich durch grippeähnliche Symptome, wie trockener Husten, Fieber, Schnupfen und Abgeschlagenheit. Auch über Atemprobleme, Halskratzen, Kopf- und Gliederschmerzen, Übelkeit, Durchfall sowie Schüttelfrost wurde berichtet. Wenden Sie sich telefonisch an Ihre Frauenärztin bzw. Ihren Frauenarzt. Durch diese/n werden Sie über alle weiteren Maßnahmen aufgeklärt. Wenden Sie sich unverzüglich und unabhängig von Symptomen telefonisch an Ihr zuständiges Gesundheitsamt (dieses können Sie hier ermitteln: https://tools.rki.de/plztool/), oder 
rufen Sie den ärztlichen Bereitschaftsdienst unter der Telefonnummer 116117. Aktuell sind noch keine Behandlungsmöglichkeiten oder Impfungen bekannt. Die Diagnose wird mit einem Abstrichbefund aus dem Mund- und Rachenbereich gestellt.

Was soll ich tun, wenn ich positiv auf das Coronavirus getestet wurde?

Wenn Sie positiv auf das Coronavirus getestet wurden, sollten Sie sich telefonisch an Ihre/n Frauenärztin/-arzt wenden, um auf Ihre Diagnose aufmerksam zu machen. Wenn Sie keine oder leichte Symptome haben und keine Risikofaktoren für Komplikationen bei Ihnen oder weiteren Personen im Haushalt vorliegen (z. B. relevante chronische Grunderkrankungen), können Sie sich unter Betreuung durch einen behandelnden Arzt zu Hause erholen. Wenn Sie schwerere Symptome haben, werden Sie möglicherweise in einem Krankenhaus behandelt.

\section{Wann sollte ich isoliert werden?}

Eine Isolation wird empfohlen, wenn:

- Sie mit jemandem in Kontakt gekommen sind, der an einer Coronavirusinfektion leidet;

- Sie ein bestimmtes Gebiet oder Land besucht haben, in denen COVID-19-Fälle vorkommen oder das als Risikogebiet ausgewiesen wurde;

- Sie Symptome haben, die auf eine Infektion mit dem Coronavirus hinweisen, und darauf warten, getestet zu werden oder auf Ihre Ergebnisse;

- Sie positiv auf das Coronavirus getestet wurden und es Ihnen empfohlen wurde, sich zu Hause zu erholen.

\section{Kriterien zur Aufhebung von Isolierungsmaßnahmen und zur Entlassung aus dem \\ Krankenhaus}

Nach aktuellem Wissensstand ist eine Entisolierung zu Hause bzw. Entlassung aus der Klinik frühestens 10 Tage nach Symptombeginn und Erfüllung ALLER folgender Kriterien vertretbar:

- Fieberfreiheit seit mind. 48 Stunden;

- Symptomfreiheit seit mind. 24 Stunden bezogen auf die akute COVID-19-Erkrankung sowie
- 2 negative SARS-CoV-2-PCR-Untersuchungen im Abstand von 24 Stunden gewonnen aus Mund-Rachen-Abstrichen.

Was soll ich tun, wenn ich aufgefordert werde, mich selbst zu isolieren?

Schwangere, denen geraten wurde, sich selbst zu isolieren, sollten im Haus bleiben und 14 Tage lang den Kontakt mit anderen vermeiden. Das RKI gibt hierzu dezidierte Empfehlungen: https://www.rki.de/DE/ Content/InfAZ/N/Neuartiges_Coronavirus/ ambulant.html

Konkret bedeutet das:

- nicht zur Schule oder zur Arbeit oder in öffentliche Bereiche gehen;

- keine öffentlichen Verkehrsmittel benutzen - bleiben Sie zu Hause und erlauben Sie keine Besuche;

- lüften Sie die Räume, in denen Sie sich befinden;

- trennen Sie sich so weit wie möglich von anderen Personen im Haushalt, indem Sie Ihre eigenen Handtücher, Geschirr und Utensilien verwenden und zu unterschiedlichen Zeiten essen;

- bitten Sie Freunde und Familie oder nutzen Sie Lieferservices, um Besorgungen für Sie zu erledigen. Die Übergabe sollte dann ohne direkten Kontakt erfolgen.

Kann ich trotzdem an meinen vorgeburtlichen Terminen teilnehmen, wenn ich mich selbst isoliert habe?

Sie sollten sich an Ihre Frauenärztin bzw. Ihren Frauenarzt wenden, um sie/ihn darüber zu informieren, dass Sie sich derzeit in Selbstisolierung für eine mögliche beziehungsweise bestätigte Coronavirusinfektion befinden. Es ist wahrscheinlich, dass routinemäßige vorgeburtliche Termine ohne Schaden für Sie oder Ihr Kind herauszögert werden, bis die Isolation endet.

Wenn Ihre Hebamme oder Ihr Arzt darauf hinweist, dass Ihr Termin nicht warten kann, werden die erforderlichen Vorkehrungen getroffen, damit Sie den Termin wahrnehmen können.

Was mache ich, wenn ich mich unwohl fühle oder mir während der Selbstisolation Sorgen um mein Baby mache? Ihr ambulant betreuender Arzt und das zuständige Gesundheitsamt sollten gemeinsam mit Ihnen und ggf. Ihrer Betreuungs- person das Vorgehen im Falle einer notfallmäßigen bzw. außerhalb der üblichen Erreichbarkeiten eintretenden Zustandsverschlechterung im Vorhinein festlegen. Das Vorgehen sollte sowohl das aufnehmende Krankenhaus, die mitzuführenden Unterlagen als auch das geeignete Transportmittel dorthin umfassen. Schwangeren wird empfohlen, keine allgemeinen Sprechstunden oder Kliniken zu besuchen, es sei denn, sie benötigen dringend eine medizinische Versorgung. Wenn Sie während Ihrer Selbstisolationsphase Bedenken hinsichtlich des Wohlbefindens Ihrer selbst oder Ihres ungeborenen Kindes haben, wenden Sie sich an Ihren Frauenarzt bzw. Ihre Frauenärztin. Wenn dort die Aufnahme in ein Krankenhaus empfohlen wird, werden schwangere Frauen gebeten, mit privaten Verkehrsmitteln zu reisen oder einen Krankenhaustransport zu arrangieren und vor dem Betreten des Krankenhauses die Klinik telefonisch zu benachrichtigen.

Wird sich die Selbstisolierung aufgrund einer vermuteten oder bestätigten Infektion mit COVID-19 darauf auswirken, wo ich gebäre?

Als Vorsichtsmaßnahme wird schwangeren Frauen mit Verdacht auf oder bestätigter Coronavirusinfektion bei Wehen empfohlen, zur Geburt eine Klinik aufzusuchen, in der das Baby kontinuierlich elektronisch überwacht und der Sauerstoffgehalt stündlich geprüft werden kann. Die kontinuierliche Überwachung des Fetus dient dazu, zu überprüfen, wie Ihr Baby auf Wehen reagiert. Da eine kontinuierliche Überwachung des Fetus nur in einer geburtshilflichen Abteilung stattfinden kann, in der Ärzte und Hebammen anwesend sind, wird nicht empfohlen, zu Hause oder in einem Geburtshaus zu gebären, in dem nur Hebammen anwesend sind.

Wird sich die Selbstisolierung aufgrund einer vermuteten oder bestätigten Infektion mit COVID-19 auf meine Geburt auswirken?

Derzeit gibt es keine Anhaltspunkte dafür, dass Sie nicht vaginal gebären können oder dass ein Kaiserschnitt sicherer ist, wenn eine Coronavirusinfektion vermutet oder bestätigt wurde. Wenn Ihre Atemwegserkrankung (Atmung) jedoch darauf hindeutet, dass eine dringende Entbindung erforderlich ist, kann eine Kaiserschnittgeburt 
empfohlen werden. Es gibt auch keine Hinweise darauf, dass Frauen mit Verdacht auf oder mit bestätigter Coronavirusinfektion keine PDA haben können. Die Verwendung von Lachgas kann jedoch die Aerosolisierung und Ausbreitung des Virus erhöhen davon wird ausdrücklich abgeraten.

Was passiert, wenn ich während meiner Selbstisolationsphase Wehen bekomme? Wenn Sie Wehen bekommen, sollten Sie Ihre Entbindungsstation um Rat fragen und sie darüber informieren, dass Sie eine Coronavirusinfektion vermuten oder bestätigt haben. Ihr Geburtsteam wird Sie über Möglichkeiten beraten.

Kann ich sicher sein, dass ich zur Geburt eine Begleitperson mit in die Klinik nehmen kann?

Derzeit wird in vielen Geburtskliniken noch eine Begleitperson zur Geburt zugelassen. Diese Einschätzung kann sich allerdings jederzeit ändern.

\section{Könnte ich meinem Baby das Coronavirus übertragen?}

Da es sich um ein neues Virus handelt, gibt es nur begrenzte Hinweise auf die Behandlung der Coronavirusinfektion bei Frauen, die gerade geboren haben. Es gibt jedoch keine Berichte darüber, dass Frauen, bei denen im 3. Schwangerschaftstrimester eine Coronavirusinfektion diagnostiziert wurde, das Virus im Mutterleib an ihre Babys weitergegeben haben. Die Datenbasis ist hier sehr gering; bei 20 Fällen wurde bei den
Neugeborenen kein Virus nachgewiesen. Alle Infektionen sind im 3. Trimenon erfolgt.

Wird mein Baby auf Coronavirus getestet? Ja, wenn zum Zeitpunkt der Geburt Ihres Babys ein Coronavirus bei Ihnen vermutet oder bestätigt wurde, wird Ihr Baby auf Coronavirus getestet.

Kann ich bei meinem Baby bleiben, wenn das Coronavirus bei mir vermutet oder bestätigt wird?

Ja, wenn das Ihr Wunsch ist. Vorausgesetzt, Ihrem Baby geht es gut und es muss nicht in der Neugeborenenstation gepflegt werden. Eine Diskussion über die Risiken und Vorteile sollte zwischen Ihnen, Ihrer Familie und den Ärztinnen bzw. Ärzten, die sich um Ihr Baby kümmern, stattfinden. Diese Empfehlung kann sich ändern, wenn sich das Wissen über das neue Virus weiterentwickelt.

\section{Kann ich mein Baby stillen?}

Ja. Derzeit fehlen evidenzbasierte Hinweise dafür, dass das Virus über die Muttermilch übertragen werden kann. Berichtet wurde lediglich über einen Einzelfall, wo in der Muttermilch die RNA (Ribonukleinsäure) des Virus festgestellt wurde und das Kind an COVID-19 erkrankte. Unklar ist jedoch, durch welchen Übertragungsweg sich der Säugling infizierte. Daher wird davon ausgegangen, dass die anerkannten Vorteile des Stillens die potenziellen Risiken einer Übertragung des Coronavirus überwiegen. Infizierte Mütter oder Verdachtsfälle sollten beim Stillen durch Hygienemaßnah- men wie gründliches Händewaschen vor und nach dem Kontakt mit dem Kind und durch das Tragen eines Mundschutzes eine Übertragung des Virus durch Tröpfcheninfektion verhindern. Diese Empfehlung kann sich ändern, wenn sich das Wissen über das neue Virus weiterentwickelt.

Wenn Sie Ihr Baby stillen möchten, werden die folgenden Vorsichtsmaßnahmen empfohlen:

- Waschen Sie Ihre Hände ausreichend lang, bevor Sie Ihr Baby, die Milchpumpe oder die Flasche berühren;

- tragen Sie eine Gesichtsmaske zum Füttern an der Brust;

- befolgen Sie nach jedem Gebrauch die Empfehlungen zur Pumpenreinigung;

- wenn Sie Ihr Baby mit Milchnahrung oder Milch füttern möchten, wird empfohlen, die Sterilisationsrichtlinien strikt einzuhalten;

- wenn Sie im Krankenhaus Muttermilch abpumpen, sollte eine spezielle Pumpe verwendet werden.

Ist eine Mutter nicht in der Lage, ihr Kind zu stillen, kann die Muttermilch auch abgepumpt werden und über eine weitere Person an den Säugling verfüttert werden. Auch hierbei sollte auf die Hygiene geachtet und Pumpe sowie Fläschchen sollten nach dem Gebrauch sterilisiert werden.

Abgestimmt mit dem Royal College of Obstetricians and Gynaecologists und der Deutschen Gesellschaft für Gynäkologie und Geburtshilfe e. V. 